\title{
Planck-scale structure of spacetime and some implications for astrophysics and cosmology ${ }^{1}$
}

\author{
Giovanni AMELINO-CAMELIA \\ Dipart. Fisica Univ. La Sapienza and Sez. Roma1 INFN \\ P.le Moro 2, I-00185 Roma, Italy
}

\begin{abstract}
I briefly review some scenarios for the role of the Planck length in quantum gravity. In particular, I examine the differences between the schemes in which quantum gravity is expected to introduce a maximum acceleration and the schemes in which the Planck length sets the minimum value of wavelengths (maximum value of momentum). I also comment on some pictures for the structure of spacetime at the Planck scale, such as spacetime discretization and spacetime noncommutativity. I stress that some of these proposals can have significant implications in astrophysics and cosmology.
\end{abstract}

\footnotetext{
${ }^{1}$ Invited talk at "Thinking, Observing and Mining the Universe", Sorrento, Italy, September 22-27, 2003
} 


\section{The Planck length as a relativistic invariant}

One of the few (perhaps the only) rather robust hint that we have about the quantum-gravity problem is that the Planck length, $L_{p} \equiv \sqrt{\hbar G / c^{3}} \sim 10^{-35} \mathrm{~m}$, should acquire a special role, and indeed in most quantum-gravity research programmes one finds or assumes that some new phenomena involve in one or another way the Planck length (or some closely-related scale, like the string length). In recent years there has been growing interest (see, e.g., Refs. 11, 2, 3, 4, 15, 6, 7, 8, 9, 10, 11, 12, 13, 14, 15, 16, 17, 18, 19, 20, 21, 22, 23 ) in establishing in which way the different scenarios for the role of the Planck length in fundamental physics affect issues that are relevant for rotation/boost transformations. In particular, there are some cases in which the fact that ordinary Lorentz boosts act nontrivially on lengths (FitzGerald-Lorentz contraction) has been found to have profound consequences.

In these notes I intend to give a general discussion of the fate of Lorentz symmetry in some illustrative examples of scenarios for the role of the Planck length in fundamental physics. And I will also briefly review some known cases in which observations in astrophysics and cosmology are being considered as possible opportunities for establishing experimentally the fate of Lorentz symmetry at the Planck scale.

My first task must be the one of describing some illustrative examples of scenarios for the role of the Planck length in fundamental physics, focusing on the implications for boost transformations.

\subsection{The Planck length as a coupling constant}

Of course, the Planck length already has a role in the present (pre-quantum-gravity) description of fundamental physics: it essentially plays the role of a coupling constant (an $\hbar, c$-rescaled square root of the gravitational coupling $G$ ). Such a role for the Planck length is of course fully compatible with the structure of ordinary Lorentz transformations. Actually, a coupling constant can be given operative meaning directly in a rest frame, and therefore, when it is operatively defined in this way, it is fully compatible with all possible laws of transformation between inertial observers.

I can clarify what I mean by "can be given operative meaning directly in a rest frame" by considering just the case of the gravitational coupling constant: I can give operative meaning to $G$ (and therefore to $L_{p}$ ) through a measurement of the force that two massive particles at rest exert on each other. Any observer that wants to establish the value of $G$ must consider two particles at rest in her reference frame. And of course, when operatively defined in this way, there is clearly no logical obstruction for the physical law that "the value of $G$ is the same for all inertial observers". In particular, this operative definition of $G$ (and $L_{p}$ ) is fully (and trivially) consistent with the structure of ordinary Lorentz transformations.

While it is legitimate to conjecture that some new role for the Planck length should be introduced at the level of quantum gravity, it remains plausible that in quantum gravity, just like in pre-quantumgravity physics, the role of the Planck length would be simply the one of a coupling constant. In particular, in String Theory the string length (which is closely related to the Planck length) is introduced as a coupling constant and appears to have some rather familiar properties of coupling constants.

\subsection{The Planck length in a maximum-acceleration framework}

An example of new role for the Planck length is provided by scenarios in which the Planck length intervenes in introducing a new maximum-acceleration limit. To my knowledge the first discussions of this type of scenario emerged in a research programme developed by Caianiello and others 24]. Actually the Caianiello programme started off without invoking a role for the Planck length, but rather seeking the introduction of a "non-universal" maximum-acceleration principle, with a different value of the maximum acceleration for different particles. Since the speed-of-light scale already provides us a velocity scale, the introduction of an acceleration scale only requires a time(/length/inverse-mass) scale, a scale that can be expressed in terms of the mass of the particle. Eventually the Caianiello programme with mass-dependent non-universal maximum acceleration encountered some difficulties, and the Planck length was then used to introduce a mass-independent universal maximum-acceleration limit.

More recently the possibility of a Planck-length-based maximum-acceleration limit has been explored in a new way [21, mostly on the basis of the fact that in Born-Infeld theory there is a maximum field strength. 
Of course, an observer-independent maximum-acceleration limit can be introduced in a way that is fully compatible with ordinary Lorentz transformations ${ }^{\mathrm{a}}$, since the acceleration of a particle does not change ${ }^{\mathrm{b}}$ under rotations and boosts.

This is somewhat analogous to the introduction of a minimum value of the modulus of the angular-momentum vector for some particles in ordinary quantum mechanics. Such a minimum angular-momentum-modulus condition can be implemented, as well known (and recently reconsidered from different perspectives in Ref. 19] and Ref. [20]), without encountering any conflict with spacerotation symmetry. Again this is due to the fact that the modulus of the angular momentum of a particle does not change under space rotations.

\subsection{The Planck length as the minimum wavelength}

While a role for the Planck length in the introduction of a coupling constant or a maximum acceleration does not require any modification of the familiar Lorentz transformations, in some cases the Planck length can be introduced in a way that requires modification of Lorentz boosts. A good example of this possibility is provided by the idea that the Planck length might set a minimum-wavelength ${ }^{\mathrm{c}}$ (and/or maximum momentum) limit.

Since ordinary boosts act nontrivially on wavelengths (by FitzGerald-Lorentz contraction) it is not possible to introduce the Planck length as an observer-independent minimum allowed value for wavelengths without modifying the structure of Special Relativity. Lorentz symmetry must be either "broken" or "deformed" as one can easily see in a specific example of minimum-wavelength scenario, the one in which the $\omega / \lambda$ frequency/wavelength dispersion relation (for a massless particle) takes the form

$$
\frac{L_{p}}{\lambda}=1-e^{-L_{p} \omega / c},
$$

The possible implications of (1) for Special Relativity can be easily analyzed in analogy with the possible implications of the relation $E=\sqrt{c^{2} p^{2}+c^{4} m^{2}}$ for Galilei-Newton Relativity. According to Galilei-Newton Relativity the relativistic-invariant relation between energy and momentum is $E=p^{2} /(2 m)$, and therefore there are only two possibilities: if the Galilei-Newton rotation/boost transformations are not modified the relation $E=\sqrt{c^{2} p^{2}+c^{4} m^{2}}$ can only hold for a single ("preferred") class of inertial observers (the "ether"); if instead the relation $E=\sqrt{c^{2} p^{2}+c^{4} m^{2}}$ is introduced as an observer-independent law then the Galilei-Newton rotation/boost transformations must necessarily be "deformed". The Lorentz rotation/boost transformations are a "deformation" of the Galilei-Newton rotation/boost transformations: they both are 6-parameter families of transformations, and in the $c^{-1} \rightarrow 0$ limit the Lorentz transformations are identical to the Galilei-Newton transformations, but in general the Lorentz prescriptions for relating observations in different reference frames differs from the corresponding Galilei-Newton prescriptions.

Analogously for what concerns (11) there are only two possibilities: if the Lorentz rotation/boost transformations are not modified the relation (11) can only hold for a single ("preferred") class of inertial observers; if instead the relation (11) is introduced as an observer-independent law then the

\footnotetext{
${ }^{a}$ But of course some nontrivial features must be introduced for the description of accelerated observers (at the General-Relativity level of analysis).

${ }^{\mathrm{b}}$ Of course, here the key point is that acceleration are invariant under Lorentz transformations. Similar schemes for introducing a length scale without modifying Lorentz symmetry can be based on other Lorentz-transformation invariants (see, e.g., Ref. 25]).

cThe possibility that the Planck length might set the minimum allowed value of wavelengths has been considered in a large number of quantum-gravity studies, but it is usually presented (see, e.g., Refs. [26, 27]) without commenting on the possibility that there might be FitzGerald-Lorentz contraction of the minimum wavelength, and without specifying how the minimum-wavelength scheme is realized for different observers, i.e. without considering the issue of whether the minimum value of wavelengths is the same for all observers or it is given by the Planck length only for one class of observers (while being subject to FitzGerald-Lorentz contraction for other observers).
} 
Lorentz boost transformations must necessarily be "deformed". The deformed Lorentz transformations would then be a 6-parameter family of symmetry transformations, which in the $L_{p} \rightarrow 0$ limit are identical to the Lorentz transformations.

For the possibility of broken Lorentz symmetry (with a "preferred" class of inertial observers) there is a long tradition in the quantum-gravity literature (see, e.g., Refs. [1, 2, 1, 8, 10, 11, and references therein). The idea of a Planck-scale deformed Lorentz symmetry, in the sense here described

(in which the boost transformations are characterized by two observer-independent scales $c$ and $L_{p}$, rather than the single invariant $c$ ), is the core feature of the more recent "Doubly Special Relativity" proposal [13, 14, 15].

\subsection{The Planck length in "quantum-gravity uncertainty principles"}

I have considered two rather different illustrative examples of situations in which the Planck scale is introduced in ways that are completely (and self-evidently) unconsequential for the analysis of Lorentz boosts, the case of the Planck scale as a coupling constant and the case of the Planck scale appearing in a maximum-acceleration limit. I also considered an illustrative example of a role for the Planck length that necessarily requires some departures from Lorentz symmetry, the case of the Planck length setting the minimum allowed value for wavelengths (which requires that Lorentz symmetry is either broken or deformed).

It is rather awkward that often in the quantum-gravity literature some novel role is attributed to the Planck length, without even commenting on the possibility of FitzGerald-Lorentz contraction, without perceiving the need to specify how the proposed new property is realized for different (at least inertial) observers. A very significant example of this incomprehensible practice is provided by part of the literature on a possible role for the Planck length in a new uncertainty principle for the measurement of lengths. It is for example often argued [28, 29, 30, 31, 32, 33, 34, 35] that there should be an uncertainty principle $\min (\delta L)=L_{p}$ for the measurement of any length $\vec{L}$, and in most cases these proposals are discussed without any comments on how this uncertainty principle should be described by different observers. The key point here is that there are important differences between the idea of a $\min (\delta L)=L_{p}$ limit for the measurement of proper lengths (the length of, say, a pencil in its rest frame) and the idea of a $\min (\delta L)=L_{p}$ limit for the measurement of all lengths, but often the proposals are formulated in the literature without even commenting on which of these two very different scenarios is being pursued.

The introduction of a $\min (\delta L)=L_{p}$ limit for the measurement of proper lengths is of course inconsequential for Lorentz transformations. It is a statement that acquires operative meaning in a rest frame, just like the type of concept of coupling constant that I considered earlier. If only the measurement of proper lengths is affected by this minimum uncertainty it is then legitimate to assume that other observers, boosted with respect to the rest frame, will find a correspondingly smaller, FitzGerald-Lorentz contracted, uncertainty.

If instead a relation of the type $\min (\delta L)=L_{p}$ should apply to all lengths (independently of the state of motion of the object), one should then contemplate the need for a deformation of the Lorentz transformations, in the sense of the mentioned "Doubly Special Relativity" idea [13, 14, 15].

\section{The Planck length in discrete or noncommutative space- times}

FitzGerald-Lorentz contraction acts on physical lengths, but a scale with dimensions of length can of course be introduced in a way that its physical role is not the one of a length. This is seen for example clearly in the case of a theoretical framework with both a maximum velocity $V_{\max }$ and a maximum acceleration $A_{\max }$ : from $V_{\max }$ and $A_{\max }$ one obtains the length scale $V_{\max }^{2} / A_{\max }$, which (has clarified in the previous section) is not subject to FitzGerald-Lorentz contraction. On the contrary, if, in an appropriate sense, the Planck length is introduced at the level of the description of the fundamental structure of spacetime it is instead natural to expect nontrivial implications for Lorentz boosts. Examples of roles for the Planck length in spacetime structure are Planck-scale spacetime discreteness and Planck-scale spacetime noncommutativity.

It is of course rather natural to explore the possibility that in a quantum gravity some spacetime observables be subject to noncommutativity and/or discretization, since the solution of the quantumgravity problem might require the introduction of some characteristic features of quantum theory in 
the description of spacetime. Most types of spacetime discretizations would be clearly incompatible with the presence of an exact continuous (Lorentz) symmetry. This is for example certainly the case 36 in approaches based on replacing the spacetime continuum with a Planck-scale-discrete network of spacetime points. But on the other hand it is clearly not true [19, 20] that by introducing any element of discretization in a space one must necessarily renounce to the presence of continuous symmetries. For example the type of discretization of angular momentum that is predicted by ordinary quantum mechanics is fully consistent 20 with invariance under space rotations. It is therefore not possible to assume a priori that any scenario for spacetime discretization considered in the quantum-gravity literature should lead to departures from Lorentz symmetry.

In particular over these past few years there has been intense investigation of the fate of Lorentz symmetry in (the flat-spacetime limit of) Loop Quantum Gravity, which (as presently understood) predicts an inherently discretized spacetime [37. Although this discretization is not simply a description of spacetime in terms of a discrete network of spacetime points (and therefore departures from Lorentz symmetry are possible, but not automatically present), arguments presented in Refs. [2, 7, 17] support the idea of broken Lorentz symmetry in Loop Quantum Gravity. But the issue is not yet fully settled. In particular, Ref. 19 presents arguments in favour of unmodified Lorentz symmetry in Loop Quantum Gravity, whereas recently Smolin, Starodubtsev and I proposed 22] (also see the related study in Ref. 23]) a mechanism such that Loop Quantum Gravity would be described at the most fundamental level as a theory that in the flat-spacetime limit admits deformed Lorentz symmetry (in the sense of Doubly Special Relativity).

Just like there are many ways in which one can introduce some element of discretization in spacetime structure, also spacetime noncommutativity can take many different forms. Most studies have focused on various parts of the following $Q_{\mu \nu}, C_{\mu \nu}^{\beta}$ parameter space $(\mu, \nu, \beta=0,1,2,3)$

$$
\left[x_{\mu}, x_{\nu}\right]=i L_{p}^{2} Q_{\mu \nu}+i L_{p} C_{\mu \nu}^{\beta} x_{\beta}
$$

where $Q$ and $C$ are dimensionless matrices. It is at this point clear, in light of several recent results, that the only way to preserve Lorentz symmetry is the choice $Q=0=C$ (i.e. the case in which there is no noncommutativity and one is back to the familiar classical commutative Minkowski spacetime). When noncommutativity is present Lorentz symmetry is usually broken, but for some special choices of the matrices $Q$ and $C$ Lorentz symmetry might be deformed, rather than broken.

We have a rather detailed understanding [38, 39, 40, 41, 42] of the way in which Lorentz symmetry is broken in the "canonical noncommutative spacetimes" [43] with $C_{\mu \nu}^{\beta}=0\left(\left[x_{\mu}, x_{\nu}\right]=i L_{p}^{2} Q_{\mu \nu}\right)$. The matrix $Q_{\mu \nu}$ transforms like a tensor in going from one inertial observer to another (the noncommutativity is observer dependent). Particles progating in these canonical spacetimes are governed by an energy/momentum dispersion relation which is $Q$ dependent and different from $E=\sqrt{c^{2} p^{2}+c^{4} m^{2}}$. And there are birefringence effects: different polarizations of light travel at different speeds, just as it happens in the study of the propagation of light in some material crystals.

An example of noncommutative spacetime in which Lorentz symmetry might be deformed rather than broken is $\kappa$-Minkowski [5, 44, 45, 46, 47, 48]:

$$
\left[x_{\mu}, x_{\nu}\right]=i L_{p}\left(\delta_{\mu}^{\beta} \delta_{\nu}^{0}-\delta_{\nu}^{\beta} \delta_{\mu}^{0}\right)
$$

(i.e. $\left.\left[x_{j}, x_{0}\right]=i L_{p} x_{j},\left[x_{m}, x_{l}\right]=0\right)$. It appears ${ }^{\mathrm{d}}$ possible to introduce this $\kappa$-Minkowski noncommutativity as an observer-independent description of spacetime, at the price of a deformation of the Lorentz transformations. Particles progating in $\kappa$-Minkowski are governed by an energy/momentum dispersion relation which is $L_{p}$ dependent (but observer independent) and different from $E=\sqrt{c^{2} p^{2}+c^{4} m^{2}}$.

\footnotetext{
${ }^{\mathrm{d}}$ Most of the properties so far uncovered for $\kappa$-Minkowski are consistent with the structure of the mentioned "Doubly Special Relativity" framework [13 14, 15]. There are however some open issues for the compatibility of $\kappa$-Minkowski with Doubly Special Relativity, mostly concerning systems of two or more particles [20].
} 


\section{Some implications for astrophysics and cosmology}

Quantum-gravity effects are extremely small, since their magnitude is typically set by some power of the ratio between the Planck scale and the wavelength of the particle under study. There are some contexts in which the theoretical predictions can be confronted with data, but in most cases it is necessary to rely on observations in astrophysics and cosmology, rather than laboratory experiments [6]. The "astrophysics of quantum gravity" is being considered also for effects that are not directly related to the issues for Lorentz symmetry that I discussed here (see, for example, the Equivalence-Principle tests considered in Refs. [49, 50]), and has been advocated in a large number of papers on the fate of Lorentz symmetry in quantum gravity. It is at this point well established that, if Lorentz symmetry is broken or deformed at the Planck scale, there are at least a handful of opportunities for controntation with data.

One of the most studied scenarios is based on a modified dispersion relation of the type (11), using the associated small wavelength dependence of the speed of photons (based on the relation $v=d \omega / d k, k \equiv 1 / \lambda)$. The wavelength dependence of the speed of photons that is induced by (11) is of order $L_{p} / \lambda$, and is therefore completely negligible in nearly all physical contexts. It is however quite significant [1, 3. for the analysis of short-duration gamma-ray bursts that reach us from cosmological distances. For a gamma-ray burst a typical estimate of the time travelled before reaching our Earth detectors is $T \sim 10^{17} \mathrm{~s}$. Microbursts within a burst can have very short duration, as short as $10^{-4} s$. Some of the photons in these bursts have energies in the $100 \mathrm{MeV}$ range and higher (and correspondingly small wavelengths). For two photons with energy difference of order $\Delta E \sim 100 \mathrm{MeV}$ an $L_{p} \Delta E$ speed difference over a time of travel of $10^{17} \mathrm{~s}$ leads to a relative timeof-arrival delay of order $\Delta t \sim \eta T L_{p} \Delta E \sim 10^{-3} s$. Such a quantum-gravity-induced time-of-arrival delay could be revealed [1, 3] upon comparison of the structure of the gamma-ray-burst signal in different energy channels, and these types of studies are planned for the next generation of gamma-ray telescopes, such as GLAST [51.

With advanced planned neutrino observatories, such as ANTARES [52, NEMO [53] and EUSO [54, it should be possible to observe neutrinos with energies between $10^{14}$ and $10^{19} \mathrm{eV}$, and according to current models 55 gamma-ray bursters should also emit a substantial amount of high-energy neutrinos. This might provide [56, 57 another opportunity for time-of-arrival analyses.

Another example of opportunity to test schemes for Planck-scale departures from Lorentz symmetry is the one of "threshold anomalies". For example, on the basis of a Planck-scale modified energy-momentum dispersion relation that at low energies takes the form $E^{2} \simeq c^{2} p^{2}+c^{4} m^{2}+L_{p} E p^{2}$ (which could be inspired by (11)), and assuming unmodified law of energy-momentum conservation (which is compatible with the modified dispersion relation in a scheme with Lorentz-symmetry breaking), one finds that a collision between a soft photon of energy $\epsilon$ and a high-energy photon of energy $E$ can produce an electron positron pair $\left(\gamma+\gamma \rightarrow e^{+}+e^{-}\right)$only if $E \geq E_{t h}$, with the threshold energy $E_{t h}$ given by [10]

$$
E_{t h} \epsilon-\eta L_{p} E_{t h}^{3} / 8 \simeq m_{e}^{2}
$$

Analogous modifications of threshold relations are found for other processes. In particular, the case of photopion production, $p+\gamma \rightarrow p+\pi$, also leads to an analogous result in the case in which the incoming proton has high energy $E$ while the incoming photon has energy $\epsilon$ such that $\epsilon \ll E$. And the photopion-production threshold is relevant for the analysis of UHE (ultra-high-energy) cosmic rays, since a characteristic feature of the expected cosmic-ray spectrum, the so-called "GZK limit", depends on the evaluation of the minimum energy required of a cosmic ray in order to produce pions in collisions with cosmic-microwave-background photons. Strong interest was generated by the observation [4, 8, 9, 10, 11, 58, 59 that a Planck-scale-modified threshold relation can lead to a significantly higher estimate of the threshold energy, resulting an upward shift of the GZK limit. This would provide a description of the observations of the high-energy cosmic-ray spectrum reported by AGASA 60, which can be interpreted as an indication of a sizeable upward shift of the GZK limit. (But I must stress here that there are other plausible theory explanations for the AGASA "cosmic-ray puzzle", and the experimental side must be further explored, since another cosmic-ray observatory, HIRES, has not confirmed the AGASA results.)

An example of application of ideas for the fate of Lorentz at the Planck scale in cosmology is given by the studies in Ref. [16. The observation that some of the frontier ideas in Planckscale-physics research can be important in cosmology has already a rather long tradition (see, e.g., Ref. 61, 62, 63, 64). In Ref. [16 it is observed that the recent proposal of Doubly Special Relativity schemes provides an opportunity for a reformulation of the "time-varying speed of light" cosmological scenario 65] (which had been previously structured relying on a preferred frame). 


\section{References}

[1] G. Amelino-Camelia, J. Ellis, N.E. Mavromatos and D.V. Nanopoulos, hep-th/9605211, Int. J. Mod. Phys. A12 (1997) 607; G. Amelino-Camelia, J. Ellis, N.E. Mavromatos, D.V. Nanopoulos and S. Sarkar, astro-ph/9712103, Nature 393 (1998) 763.

[2] R. Gambini and J. Pullin, Phys. Rev. D59 (1999) 124021.

[3] S.D. Biller et al, Phys. Rev. Lett. 83 (1999) 2108.

[4] T. Kifune, Astrophys. J. Lett. 518 (1999) L21.

[5] G. Amelino-Camelia and S. Majid, hep-th/9907110, Int. J. Mod. Phys. A15 (2000) 4301.

[6] G. Amelino-Camelia, "Are we at the dawn of quantum-gravity phenomenology?", gr-qc/9910089, Lect. Notes Phys. 541 (2000) 1; "Quantum-gravity phenomenology: status and prospects", gr-qc/0204051, Mod. Phys. Lett. A17 (2002) 899.

[7] J. Alfaro, H.A. Morales-Tecotl and L.F. Urrutia, Phys. Rev. Lett. 84 (2000) 2318.

[8] R. Aloisio, P. Blasi, P.L. Ghia and A.F. Grillo, Phys. Rev. D62 (2000) 053010.

[9] R.J. Protheroe and H. Meyer, Phys. Lett. B493 (2000) 1.

[10] G. Amelino-Camelia and T. Piran, astro-ph/0008107, Phys. Rev. D64 (2001) 036005; G. Amelino-Camelia, gr-qc/0012049, Nature 408 (200) 661.

[11] T. Jacobson, S. Liberati and D. Mattingly, hep-ph/0112207.

[12] G. Amelino-Camelia, gr-qc/0107086, Phys. Lett. B528 (2002) 181; G. Amelino-Camelia, M. Arzano, Y.J. Ng, T. Piran, H. Van Dam, hep-ph/0307027.

[13] G. Amelino-Camelia, gr-qc/0012051, Int. J. Mod. Phys. D11 (2002) 35; hep-th/0012238, Phys. Lett. B510 (2001) 255; gr-qc/0207049, Nature 418 (2002) 34; gr-qc/0210063, Int. J. Mod. Phys. D11 (2002) 1643.

[14] J. Kowalski-Glikman, hep-th/0102098, Phys. Lett. A286 (2001) 391; R. Bruno, G. AmelinoCamelia and J. Kowalski-Glikman, hep-th/0107039, Phys. Lett. B522 (2001) 133; G. AmelinoCamelia, D. Benedetti, F. D'Andrea, hep-th/0201245, Class. Quantum Grav. 20 (2003) 5353; J. Kowalski-Glikman and S. Nowak, hep-th/0204245.

[15] J. Magueijo and L. Smolin, gr-qc/0207085, Phys. Rev. D67 (2003) 044017; gr-qc/0305055.

[16] S. Alexander and J. Magueijo, hep-th/0104093 S. Alexander, R. Brandenberger and J. Magueijo, hep-th/0108190, Phys.Rev. D67 (2003) 081301.

[17] T. Thiemann, gr-qc/0110034.

[18] T.J. Konopka and S.A. Major, New J. Phys. 4 (2002) 57.

[19] C. Rovelli and S. Speziale, gr-qc/0205108.

[20] G. Amelino-Camelia, gr-qc/0205125; gr-qc/0309054.

[21] F.P. Schuller, M.N.R. Wohlfarth and T.W. Grimm, hep-th/0211264, Class. Quantum Grav. 20 (2003) 3947; F.P. Schuller and Hendryk Pfeiffer, hep-th/0307247.

[22] G. Amelino-Camelia, L. Smolin and A. Starodubtsev, hep-th/0306134

[23] L. Freidel, J. Kowalski-Glikman and L. Smolin, hep-th/0307085.

[24] E.R. Caianiello, M. Gasperini and G. Scarpetta, Nuovo Cim. 105B (1990) 259; E.R. Caianiello, Riv. Nuovo Cim. 15 (1992) 1. 
[25] A. Kempf, gr-qc/0310035.

[26] A. Kempf, G. Mangano and R.B. Mann, Phys. Rev. D52 (1995) 1108.

[27] D.V. Ahluwalia, Phys. Lett. A275 (2000) 31.

[28] C.A. Mead, Phys. Rev. 135 (1964) B849.

[29] T. Padmanabhan, Class. Quantum Grav. 4 (1987) L107.

[30] S. Doplicher, K. Fredenhagen and J.E. Roberts, Phys. Lett. B331 (1994) 39.

[31] D.V. Ahluwalia, Phys. Lett. B339 (1994) 301.

[32] Y.J. Ng and H. Van Dam, Mod. Phys. Lett. A9 (1994) 335.

[33] G. Amelino-Camelia, gr-qc/9603014, Mod. Phys. Lett. A9 (1994) 3415; gr-qc/9603013, Mod. Phys. Lett. A11 (1996) 1411.

[34] L.J. Garay, Int. J. Mod. Phys. A10 (1995) 145.

[35] F. Scardigli and R. Casadio, hep-th/0307174, Class. Quantum Grav. 20 (2003) 3593.

[36] G. 't Hooft, Class. Quantum Grav. 13 (1996) 1023.

[37] C. Rovelli and L. Smolin, Nucl. Phys. B442 (1995) 593.

[38] A. Matusis, L. Susskind and N. Toumbas, hep-th/0002075, JHEP 0012 (2000) 002.

[39] A. Anisimov, T. Banks, M. Dine and M. Graesser hep-ph/0106356.

[40] G. Amelino-Camelia, L. Doplicher, S. Nam and Y.-S. Seo, hep-th/0109191, Phys. Rev. D67 (2003) 085008; G. Amelino-Camelia, G. Mandanici and K. Yoshida, hep-th/0209254.

[41] C.E. Carlson, C.D. Carone and R.F. Lebed, hep-ph/0209077, Phys. Lett. B549 (2002) 337.

[42] N.R. Douglas and N.A. Nekrasov, Rev. Mod. Phys. 73 (2001) 977.

[43] J. Madore, S. Schraml, P. Schupp and J. Wess, hep-th/0001203, Eur. Phys. J. C16 (2000) 161.

[44] S. Majid and H. Ruegg, Phys. Lett. B334 (1994) 348.

[45] J. Lukierski, H. Ruegg and W.J. Zakrzewski Ann. Phys. 243 (1995) 90.

[46] P. Kosinski, J. Lukierski and P. Maslanka, Czech. J. Phys. 50 (2000) 1283.

[47] G. Amelino-Camelia and M. Arzano, hep-th/0105120, Phys. Rev. D65 (2002) 084044; Giovanni Amelino-Camelia, Francesco D'Andrea, Gianluca Mandanici, hep-th/0211022, JCAP 0309 (2003) 006; A. Agostini, G. Amelino-Camelia and M. Arzano, gr-qc/0207003; A. Agostini, G. AmelinoCamelia, F. D'Andrea, hep-th/0306013.

[48] M. Dimitrijevic, L. Jonke, L. Moller, E. Tsouchnika, J. Wess and M. Wohlgenannt, hep-th/0307149.

[49] M. Gasperini, Phys. Rev. D38 (1988) 2635.

[50] G.Z. Adunas, E. Rodriguez-Milla and D.V. Ahluwalia, Gen. Rel. Grav. 33 (2001) 183.

[51] J.P. Norris, J.T. Bonnell, G.F. Marani, J.D. Scargle, astro-ph/9912136 A. de Angelis, astro-ph/0009271.

[52] Information on ANTARES available at antares.in2p3.fr

[53] Information on NEMO available at www.lal.in2p3.fr/recherche/nemo 
[54] Information on EUSO available at euso.riken.go.jp

[55] P. Meszaros, S. Kobayashi, S. Razzaque, B. Zhang, astro-ph/0305066.

[56] J.R. Ellis, N.E. Mavromatos, D.V. Nanopoulos and G. Volkov, gr-qc/9911055, Gen. Rel. Grav. 32 (2000) 1777; S. Choubey, S.F. King, hep-ph/0207260.

[57] G. Amelino-Camelia, gr-qc/0305057.

[58] J. Alfaro and G. Palma, hep-th/0208193 Phys. Rev. D67 (2003) 083003.

[59] O. Bertolami, hep-ph/0301191.

[60] M. Takeda et al, Phys. Rev. Lett. 81 (1998) 1163.

[61] R. Brustein, M. Gasperini, M. Giovannini and G. Veneziano, Phys. Lett. B361 (1995) 45; R. Brustein, gr-qc/9810063; G. Veneziano, hep-th/9902097; M. Gasperini, hep-th/9907067.

[62] Q.-G. Huang, M. Li, hep-th/0304203, JHEP 0306 (2003) 014; astro-ph/0311378.

[63] S. Cremonini, hep-th/0305244, Phys. Rev. D68 (2003) 063514.

[64] R. Casadio and L. Mersini, hep-th/0208050; G.L. Alberghi, R. Casadio and A. Tronconi, gr-qc/0303035.

[65] J.W. Moffat Int. J. Mod. Phys. D2 (1993) 351; J.D. Barrow, Phys. Rev. D59 (1999) 043515; A. Albrecht and J. Magueijo, Phys. Rev. D59 (1999) 043516; J. Magueijo, astro-ph/0305457, Rept. Prog. Phys. 66 (2003) 2025. 